\title{
ON NONOSCILLATORY LINEAR DIFFERENTIAL EQUATIONS OF SECOND ORDER
}

\author{
PHILIP HARTMAN ${ }^{1}$
}

ABSTRACT. For the equation $y^{\prime \prime}+q(t) y=0$, we derive necessary conditions for nonoscillation in terms of "general means" of $Q(t)=\int_{0}^{t} q d s$.

1. Main theorem. The object of this note is to obtain necessary conditions for

$$
y^{\prime \prime}+q(t) y=0
$$

$q \in C^{0}[0, \omega)$, to be nonoscillatory at $t=\omega(<\infty)$. The result generalizes [2] (cf. [3, §7, pp. 362-369]) involving the case $\omega=\infty$ and the arithmetic mean

$$
t^{-1} \int_{0}^{t} Q(s) d s, \text { where } Q(t)=\int_{0}^{t} q(s) d s .
$$

A generalization of [2] was given by Coles and Willett in which $\omega=\infty$, and the arithmetical mean was replaced by a more general mean

$$
(K Q)(t)=\int_{0}^{t} K(t, s) Q(s) d s,
$$

where $K(t, s)$ is the kernel $K_{n}(t, s)=K_{n}\left(t, s ; \phi_{1}, \ldots, \phi_{n}\right)$,

$$
\begin{gathered}
K_{n}(t, s)=\phi_{1}(s) \int_{s<s_{2}<} \underset{s_{n}<t}{\ldots} \int \phi_{2}\left(s_{2}\right) \cdots \phi_{n}\left(s_{n}\right) d s_{2} \cdots d s_{n} / k_{n}(t), \\
\quad \int_{0}^{t} K_{n}(t, s) d s=1,
\end{gathered}
$$

depending on nonnegative functions $\phi_{1}, \ldots, \phi_{n}$; cf. [1], [5] and references there, and see $\$ 4$ below. The essential arguments in these papers were the same as in [2], but they also involved many formal calculations with iterated integrals which seemed to have nothing to do with (1.1). In this paper, we show that the methods of [2] can be used for very general "means" (1.2) and, in fact, the object in part is to state suitable conditions on the kernel $K(t, s)>0$ for which the arguments of [2] are applicable. Roughly, the conditions imposed on $K$ will include the following three types: first, a generalization of the condition that the map $Q \mapsto K Q$ in (1.2) is regular in the

Received by the editors September 16, 1976.

AMS (MOS) subject classifications (1970). Primary $34 \mathrm{Cl0}$.

${ }^{1}$ This study was supported by NSF Grant MPS75-15733. 
sense of (Toeplitz) summation theory [cf. (a), ( $\left.\mathrm{b}^{*}\right)$ below]; second, that this map is a bounded integral operator from the Banach space $\left(L^{2 / \sigma} \cap C^{9}\right)[0, \omega)$ to the Banach space $\left(L_{0}^{\infty} \cap C^{9}\right)[0, \omega)$ for some $\sigma, 1<\sigma<2$, [cf. $\left(\mathrm{c}_{\sigma}\right)$ below] and, third, a quite technical condition given in (d) below. Our main result will be stated as a sufficient condition for $\left(\mathrm{A}_{\tau}\right)$ :

$\left(\mathrm{A}_{\tau}\right)$ Let $\tau$ be fixed, $1 \leqslant \tau \leqslant 2$. Let $K(t, s) \geqslant 0$ be continuous for $0 \leqslant s<$ $t, 0<t<\omega(\leqslant \infty)$. We say that the kernel $K$ has property $\left(\mathrm{A}_{\tau}\right)$ if the following holds: If $q(t) \in C^{0}[0, \omega)$, then a necessary condition for (1.1) to be nonoscillatory at $t=\omega$ is either that

$$
\liminf _{t \rightarrow \omega} \int_{0}^{t} K(t, s) Q(s) d s=-\infty,
$$

or that there exists a constant $C$ such that

$$
\int_{0}^{t} K(t, s)|C-Q(s)|^{\tau} d s \rightarrow 0 \quad \text { as } t \rightarrow \omega .
$$

Furthermore, when (1.1) is nonoscillatory, then (1.5) holds if and only if one and/or every solution $y \not \equiv 0$ of (1.1) satisfies

$$
\int^{\omega}\left(y^{\prime} / y\right)^{2} d t<\infty
$$

REMARK. We do not gain generality by replacing (1.1) by an equation of the form $\left(P(t) y^{\prime}\right)^{\prime}+R(t) y=0, P(t)>0$, since the latter can be reduced to (1.1) by the change of independent variables $t \rightarrow u, d u=d t / P(t)$, and $K(t, s)$ replaced by $K(t, s) P(s)$.

We use the letter $K$ for both the kernel $K(t, s)$ and the corresponding integral operator $Q \mapsto K Q$ in (1.2). In what follows, we always assume that $K(t, s) \geqslant 0$ is continuous for $0 \leqslant s \leqslant t, 0<t<\omega$. We list some other conditions on $K$ which will be assumed from time to time:

$$
(K Q)(t) \rightarrow 0, \quad t \rightarrow \omega, \quad \text { if } Q \in\left(L_{0}^{\infty} \cap C^{0}\right)[0, \omega),
$$

i.e., the operator $K$ is a bounded map on the Banach space $\left(L_{0}^{\infty} \cap C^{9}\right)[0, \omega)$.

$$
\begin{gathered}
(K Q)(t) \rightarrow \infty, \quad t \rightarrow \omega, \quad \text { if } C^{0}[0, \omega) \ni Q(t) \rightarrow \infty, t \rightarrow \omega . \\
(K Q)(t) \rightarrow 0, \quad t \rightarrow \omega, \quad \text { if } Q \in\left(L^{2 / \sigma} \cap C^{0}\right)[0, \omega)
\end{gathered}
$$

for some fixed $\sigma, 1 \leqslant \sigma \leqslant 2$; i.e., the map $K$ is a bounded operator from the Banach space $\left(L^{2 / \sigma} \cap C^{9}\right)[0, \omega)$ to the Banach space $\left(L_{0}^{\infty} \cap C^{9}\right)[0, \omega)$ for some $\sigma, 1 \leqslant \sigma \leqslant 2$.

(d) Assume that there exist functions $0<k(t) \in C^{0}[0, \omega), 0 \leqslant m(t) \in$ $C^{0}[0, \omega)$, a kernel $K_{0}(t, s) \geqslant 0$ continuous for $0 \leqslant s \leqslant t, 0<t<\omega$, and a constant $\alpha, 0 \leqslant \alpha \leqslant 2$, with the following properties:

$$
\kappa(t, s)=\frac{\partial}{\partial t} \int_{s}^{t} k(t) K(t, s) d s \geqslant 0
$$

exists and is continuous, $0 \leqslant s \leqslant t, 0<t<\omega$;

$$
K^{2-\alpha}(t, s) / K_{0}(t, s) \text { is continuous for } 0 \leqslant s \leqslant t, 0<t<\omega ;
$$




$$
\int^{\omega} m(t) d t=\infty \text { or }(\infty>) \limsup k(t) \int_{t}^{\omega} m(t) d t>0,
$$

and, finally, (1.7) satisfies for $s$ near $\omega, s<t<\omega$,

$$
\kappa(t, s)>k^{2}(t) m(t)\left(\int_{0}^{t} K^{2-\alpha}(t, u) / K_{0}(t, u) d u\right) K^{\alpha}(t, s) K_{0}(t, s) .
$$

ReMARKs. We give examples and illustrations of these conditions in \$4, but we make some simple comments here. Condition (a) holds if and only if $K$ satisfies the two conditions

$$
\int_{0}^{t} K(t, s) d s=O(1) \text { as } t \rightarrow \omega,
$$

$\left(\mathrm{a}_{2}\right) \quad \int_{0}^{T} K(t, s) d s=o(1), \quad t \rightarrow \omega$, for fixed $T, 0<T<\omega$,

while $\left(a_{2}\right)$ is implied by

$$
K(t, s) d s=o(1), \quad t \rightarrow \omega, \quad \text { uniformly for } 0<s<T .
$$

When (a), i.e., $\left(a_{1}\right)-\left(a_{2}\right)$, holds, condition $(b)$ is satisfied if

$$
\int_{0}^{t} K(t, s) d s>c>0 \text { for } t \text { near } \omega, c=\text { const. }
$$

Also, when $\left(a_{2}\right)$ holds, then condition $\left(c_{o}\right)$ is equivalent to

$$
K(t, s)=O(1), \quad s \rightarrow \omega, \text { uniformly in } t \geq s, \sigma=2,
$$

$$
\int_{s}^{t}|K(t, u)|^{2 /(2-\sigma)} d u=O(1), \quad s \rightarrow \omega,
$$

$$
\text { uniformly in } t>s, 1<\sigma<2 \text {. }
$$

If $k \in C^{1}$, then (1.9) holds, when either

$\left(1.9^{*}\right) \int^{\omega} m(t) d t=\infty$ or $k(t) \rightarrow \infty$ and $k^{\prime} / k^{2}=O(m)$ as $t \rightarrow \omega$.

Also, if $K_{0}(t, s)=\kappa(t, s)$ satisfies (1.8) with $\alpha=0$, then (1.10) holds with

$$
m(t)=1 / k^{2}(t) \int_{0}^{t}\left[K^{2}(t, u) / \kappa(t, u)\right] d u .
$$

THEOREM. Let $q \in C 90, \omega)$ be such that (1.1) is nonoscillatory at $t=\omega$. (i) If $K$ satisfies (a) and $\left(\mathrm{c}_{\sigma}\right)$, and (1.6) holds for some solution $y \neq 0$ of (1.1), then (1.5) holds for $1<\tau<\sigma$, with

$$
C=y^{\prime}(a) / y(a)+Q(a)-\int_{a}^{\omega}\left(y^{\prime} / y\right)^{2} d s
$$

if $y(t) \neq 0$ for $(0<) a \leqslant t<\omega$; in particular,

$$
\liminf _{t \rightarrow \omega} \int_{0}^{t} K(t, s) Q(s) d s>-\infty .
$$

(ii) If $K$ satisfies (a), (b), (d) and if (1.14) holds, then every solution $y \neq 0$ of (1.1) satisfies (1.6). 
If $\left(\mathrm{b}^{*}\right)$ also holds in (i), then of course

$$
C=\lim _{t \rightarrow \omega} \int_{0}^{t} K(t, s) Q(s) d s / \int_{0}^{t} K(t, u) d u .
$$

The proof of the theorem follows [2]. It obviously implies

Corollary. Conditions (a), (b), (c $\left.\mathbf{c}_{\sigma}\right),(\mathrm{d})$ imply $\left(\mathbf{A}_{\mathrm{o}}\right)$.

2. Proof of (i). Let $y \neq 0$ be a solution of (1.1) satisfying (1.6). We shall verify (1.5) for $\tau=\sigma$. The cases $1 \leqslant \tau<\sigma$ then follow from Hölder's inequality and $\left(a_{1}\right)$, while $(1.14)$ is implied by the case $\tau=1$ of $(1.5)$ and $\left(a_{1}\right)$. Also (1.13) will be clear from the proof.

Suppose that $y \neq 0$ for $t \geqslant a(\geqslant 0)$, so that $r=y^{\prime} / y$ satisfies the Riccati equation

$$
r^{\prime}+r^{2}+q(t)=0
$$

for $t \geqslant a$. A quadrature gives

$$
r(s)+\int_{a}^{s} r^{2}(u) d u=r(a)-Q(s)+Q(a) .
$$

In view of (1.6), we can write this as

$$
r(s)-\int_{s}^{\omega} r^{2}(u) d u=C-Q(s),
$$

where $C$ is given by (1.13). Consequently, by (2.3),

so that $K \geqslant 0$ gives

$$
|C-Q(s)|^{\sigma}<2^{\sigma} r^{\sigma}(s)+2^{\sigma}\left(\int_{s}^{\omega} r^{2}(u) d u\right)^{\sigma},
$$

$$
\begin{aligned}
\int_{a}^{t} K(t, s)|C-Q(s)|^{\sigma} d s \\
<2^{\sigma} \int_{a}^{t} K(t, s) r^{\circ}(s) d s+2^{\circ} \int_{a}^{t} K(t, s)\left(\int_{s}^{\omega} r^{2}(u) d u\right)^{\circ} d s .
\end{aligned}
$$

By (a), the last term on the right tends to 0 as $t \rightarrow \omega$. Also, by $\left(c_{\sigma}\right)$, the first term tends to 0 as $t \rightarrow \omega$. This proves (i).

3. Proof of (ii). Suppose (ii) is false, so that (1.6) fails for some solution $y \neq 0$ of (1.1). Let $t=a$ be fixed, sufficiently near to $\omega$, so that, in particular, $y(t) \neq 0$ for $t>a$. Using (2.2),

(3.1) $\int_{a}^{t} K(t, s) r(s) d s+S(t)=c(t, a)-\int_{0}^{t} K(t, s) Q(s) d s, \quad t>a$,

where we put

$$
\begin{gathered}
S(t)=\int_{a}^{t} K(t, s)\left(\int_{a}^{s} r^{2}(u) d u\right) d s \\
c(t, a)=[r(a)+Q(a)] \int_{a}^{t} K(t, s) d s+\int_{0}^{a} K(t, s) Q(s) d s .
\end{gathered}
$$


Since (1.6) does not hold, (b) implies that

$$
S(t) \rightarrow \infty \text { as } t \rightarrow \omega .
$$

Note that $c(t, a)$ is bounded as $t \rightarrow \omega$ by assumption $\left(a_{1}\right)$. Hence (1.14) implies that the right side of (3.1) is bounded from above as $t \rightarrow \omega$, so that

$$
-\int_{a}^{t} K(t, s) r(s) d s>S(t) / 2 \text { for } t \text { near } \omega .
$$

For a fixed constant $\alpha$, Schwarz's inequality gives

$$
\begin{aligned}
& \left|\int_{a}^{t} K(t, s) r(s) d s\right|^{2} \\
& <\left(\int_{a}^{t}\left[K^{2-\alpha}(t, u) / K_{0}(t, u)\right] d u\right)\left(\int_{a}^{t} K^{\alpha}(t, s) K_{0}(t, s) r^{2}(s) d s\right),
\end{aligned}
$$

whenever the right side is meaningful. The last two inequalities show that

$$
\begin{gathered}
4 k^{2}(t)\left(\int_{a}^{t}\left[K^{2-\alpha}(t, u) / K_{0}(t, u)\right] d u\right)\left(\int_{a}^{t} K^{\alpha}(t, s) K_{0}(t, s) r^{2}(s) d s\right) \\
>k^{2}(t) S^{2}(t) .
\end{gathered}
$$

By Fubini's theorem, (3.2) can be rewritten

$$
S(t)=\int_{a}^{t}\left[\int_{s}^{t} K(t, u) d u\right] r^{2}(s) d s
$$

Hence, by (1.7),

$$
[k(t) S(t)]^{\prime}=\int_{a}^{t} \kappa(t, s) r^{2}(s) d s
$$

so that (1.10) and (3.7) imply

$$
4[k(t) S(t)]^{\prime}>m(t)[k(t) S(t)]^{2} \text { for } t \text { near } \omega
$$

(if $t=a$ has been fixed sufficiently near $\omega$ ). By a quadrature, for $t$ near $\omega$,

$$
4 / S(t)>k(t) \int_{t}^{u} m(s) d s \quad \text { if } t<u<\omega .
$$

This contradicts the first alternative in (1.9) if $u \rightarrow \omega$; and, by (3.4), contradicts the second alternative if $u \rightarrow \omega$ and then $t \rightarrow \omega$. This proves (ii).

4. Remarks and examples. The kernel $K(t, s)=1 / t$ for $0<s<t, t>0$, $\omega$ $=\infty$ of [2] satisfies (a), (b), ( $\left.\mathrm{c}_{2}\right)$, (d) with $k(t)=t, m(t)=1 / t, K_{0} \equiv 1$, and $\alpha=1$.

EXAMPLE 1. More generally, let $0<\phi(t) \in C^{0}[0, \omega)$,

$$
K(t, s)=\phi(t-s) / k(t), \quad \text { where } k(t)=\int_{0}^{t} \phi(s) d s .
$$

Thus $\left(a_{1}\right)$ and $\left(b^{*}\right)$ are satisfied, and $\left(a_{2}\right)$ is equivalent to

$$
\int_{t-T}^{t} \phi(s) d s / \int_{0}^{t} \phi(u) d u \rightarrow 0 \text { as } t \rightarrow \omega, 0<T<\omega .
$$


Note that

$$
\kappa(t, s)=\frac{\partial}{\partial t} \int_{s}^{t} \phi(t-u) d u=\frac{\partial}{\partial t} \int_{0}^{t-s} \phi(u) d u=\phi(t-s) .
$$

Hence, if $\alpha=1$ and $K_{0} \equiv 1$, then (1.10) holds with

$$
m(t)=1 / k(t)=1 / \int_{0}^{t} \phi(u) d u,
$$

and so, (1.9) holds if either

$$
\int^{\omega}\left[d t / \int_{0}^{t} \phi(u) d u\right]=\infty \text { or }
$$

$$
\limsup _{t \rightarrow \omega}\left(\int_{0}^{t} \phi(s) d s\right) \int_{t}^{\omega}\left[d u / \int_{0}^{u} \phi(r) d r\right]>0 .
$$

This holds if either

$$
\int^{\omega}\left[d t / \int_{0}^{t} \phi(u) d u\right]=\infty \text { or }
$$

$$
\int^{\omega} \phi d s=\infty \text { and } \phi(t)=O\left(\int_{0}^{t} \phi d u\right) \text { as } t \rightarrow \omega .
$$

Also, (1.11), hence ( $\left.c_{2}\right)$, holds if

$$
\phi(u)=O\left(\int_{0}^{u+s} \phi d v\right), \quad s \rightarrow \omega, \quad \text { uniformly for } 0<u<\omega,
$$

and (1.12), hence $\left(c_{\sigma}\right), 1<\sigma<2$, holds if

$$
\int_{0}^{u} \phi^{2 /(2-\sigma)} d v=O\left(\int_{0}^{u+s} \phi d v\right)^{2 /(2-\sigma)}, s \rightarrow \omega
$$

$$
\text { uniformly for } 0<u<\omega \text {. }
$$

EXAMPLE 2. Let $n>1$ and, if $n=1$, replace all ( $n-1)$-fold integrals below by 1 . Let $0<\phi_{1}, \ldots, \phi_{n} \in C^{9}[0, \omega)$, and consider the kernel in (1.3), i.e.,

$$
K(t, s)=L(t, s) / k(t)
$$

where

$$
L(t, s)=\phi_{1}(s) \int_{s<s_{2}<\cdots<s_{n}<t} \underset{\ldots}{\ldots} \phi_{2}\left(s_{2}\right) \cdots \phi_{n}\left(s_{n}\right) d s_{2} \cdots d s_{n},
$$

$$
k(t)=\int_{0}^{t} L(t, s) d s=\int_{0<s_{1}<\cdots<s_{n}<t} \underset{\int}{\ldots} \phi_{1}\left(s_{1}\right) \cdots \phi_{n}\left(s_{n}\right) d s_{1} \cdots d s_{n} .
$$

Then $\left(a_{1}\right),\left(b^{*}\right)$ hold, and $\left(a_{2}^{*}\right)$ is equivalent to

$$
L(t, s)=o(k(t)) \text { as } t \rightarrow \omega \text {, uniformly for } 0 \leqslant s \leqslant T \text {, }
$$

$0<T<\omega$ fixed. By repeated applications of L'Hôpital's rule, (4.11) holds if

$$
k_{m}(t) \rightarrow \infty \quad \text { as } t \rightarrow \omega \text { for } m=1, \ldots, n,
$$


where $k=k_{n}$ in (4.10). Note that

$$
\kappa(t, s)=\phi_{n}(t) \int_{s<s_{1}<\cdots<s_{n-1}<t} \ldots \int \phi_{1}\left(s_{1}\right) \cdots \phi_{n-1}\left(s_{n-1}\right) d s_{1} \cdots d s_{n-1} .
$$

If $\alpha=1$ and $K_{0}=1$, then (1.10) in (d) holds for $T<s<t<\omega$ if

$$
m(t)=\left\{\sup _{T<s<t}[\kappa(t, s) / L(t, s)]\right\} / k(t) .
$$

If $\alpha=0$ and $K_{0}=\kappa$, then (1.10) holds if

$$
m(t)=1 / \int_{0}^{t}\left[L^{2}(t, u) / \kappa(t, u)\right] d u,
$$

by the remark concerning $\left(1.10^{*}\right)$. Also, (1.11), hence $\left(c_{2}\right)$, holds if (4.11) and

$$
L(t, s)=O(k(t)), \quad s \rightarrow \omega, \text { uniformly in } t>s,
$$

and (1.12), hence ( $\left.c_{\sigma}\right), 1<\sigma<2$, holds if (4.11) and

(4.17) $\int_{s}^{t}[L(t, u)]^{2 /(2-\sigma)} d u=O\left(k^{2 /(2-\sigma)}(t)\right), \quad s \rightarrow \omega$, uniformly in $t>s$.

Consider the case $n=1$ and let $\phi=\phi_{1}$. If $\phi(t)<c_{0}$, then (4.14) can be replaced by

$$
m(t)=\phi(t) / c_{0} \int_{0}^{t} \phi(s) d s,
$$

so that the first alternative in (1.9) holds by $\left(4.12_{n}\right)$. Without any assumption of boundedness, (4.15) is

$$
m(t)=\phi(t) / \int_{0}^{t} \phi^{2}(s) d s
$$

and (1.9) becomes

$$
\begin{gathered}
\int^{\omega}\left[\phi(t) / \int_{0}^{t} \phi^{2} d u\right] d t=\infty \text { or } \\
\limsup _{t \rightarrow \omega}\left(\int_{0}^{t} \phi d s\right) \int_{t}^{\omega}\left[\phi(u) / \int_{0}^{u} \phi^{2} d v\right] d u>0
\end{gathered}
$$

which is satisfied if $\left(4.12_{n}\right)$ and

$$
\int^{\omega}\left[\phi(t) / \int^{t} \phi^{2} d u\right] d t=\infty \quad \text { or } \quad \int_{0}^{t} \phi^{2} d s=O\left(\int_{0}^{t} \phi d u\right)^{2} \quad \text { as } t \rightarrow \omega
$$

Finally, (4.16), (4.17) reduce, respectively, to

$$
\begin{gathered}
\phi(t)=O\left(\int_{0}^{t} \phi d u\right) \text { as } t \rightarrow \omega, \\
\int_{s}^{t} \phi^{2 /(2-\sigma)} d u=O\left(\int_{0}^{t} \phi d u\right)^{2 /(2-\sigma)}, s \rightarrow \omega, \text { uniformly in } t>s .
\end{gathered}
$$


The above conditions seem to be weaker than those imposed by Coles and Willett [1]. For example, when $n=2,(4.15)$ becomes

$$
m(t)=\phi_{2}(t) / \int_{0}^{t}\left[\phi_{1}^{2}(s)\left(\int_{s}^{t} \phi_{2} d u\right)^{2} /\left(\int_{s}^{t} \phi_{1} d u\right)\right] d s
$$

which is required to satisfy (1.9). In Coles and Willett [1], the analogous function is

$$
\tilde{m}(t)=\phi_{2}(t) / \int_{0}^{t} \phi_{1}^{2}(s)\left[\int_{s}^{t} \phi_{2}^{2}(u) d u / \phi_{1}(u)\right] d s .
$$

By Schwarz's inequality, $m(t) \geqslant \tilde{m}(t)$, so that (1.9) is weaker than the corresponding condition in [1]. Furthermore (4.17), with $\sigma=1$, is a weaker condition than the corresponding one in [1].

EXAMPLE 3 (ADDED 2/14/77). Using the procedure of [2], Macki and Wong [4] consider necessary conditions for (1.1) to be nonoscillatory [in fact, for analogues of $\left(A_{\tau}\right)$ ] in which (1.6) is replaced by

$$
\int^{\omega} P\left(z^{\prime} / z\right)^{2} d t, \quad \text { where } z=y / P^{1 / 2},
$$

$\omega=\infty, 0<P \in C^{1}[0, \omega), Q$ in (1.2) and (1.4)-(1.5) is replaced by

$$
Q(t)=\int_{0}^{t} P q d s-\int_{0}^{t}\left(P^{\prime} / 2 P^{1 / 2}\right)^{2} d s+P^{\prime}(t) / 2
$$

and $K$ is a kernel of the type (1.3) in Example 2 with $n=1$. Their results are included in the theorem above after suitable changes of variables; $c f$. the remark following (1.6). Suppose for a moment that $P \in C^{2}$ and make the change of dependent variables $y=z P^{1 / 2}$, so that (1.1) becomes $\left(P z^{\prime}\right)^{\prime}+R z$ $=0$, or

$$
d^{2} z / d u^{2}+R z=0, \quad \text { where } R=q P+P^{1 / 2}\left(P^{1 / 2}\right)^{\prime \prime}, 0 \leqslant u<\omega^{*},
$$
$u=u(t)$ is a new independent variable, $d u=d t / P, u(0)=0, \omega^{*}=u(\omega) \leqslant$ $\infty$. Actually, the existence of $P^{\prime \prime}$ is not needed, for the proof does not involve the equation (4.21) nor the corresponding Riccati equation, but the integrated form of the latter [cf. (2.2)],

$$
r(u)+\int_{a}^{u} r^{2}(\sigma) d \sigma=c-Q(t(u))
$$

where $Q$ is given by (4.20), $t(u)$ is the inverse of $u=u(t)$, and $r(u)=$ $z^{-1}(d z / d u)=P z^{\prime} / z$ at $t=t(u)$; cf. [4] for another derivation of (4.22). Thus the analogue of the theorem holds if the kernel $K(t(v), t(u)) P(t(u))$ satisfies the corresponding conditions with $0 \leqslant s \leqslant t, 0<t<\omega$ replaced by $0<u<v, 0<v<\omega^{*}$, and (1.6) by (4.19).

Macki and Wong also formulated conditions analogous to (a) and (c), with $\sigma=2$, for their kernel. 


\section{REFERENCES}

1. W. J. Coles and D. Willeth, Summability criteria for ascillation of second order linear differential equations, Ann. Mat. Pura Appl. 79 (1968), 391-398.

2. P. Hartman, On non-ascillatory linear differential equations of second order, Amer. J. Math. 74 (1952), 389-400.

3. Ordinary differential equations, S. M. Hartman, Baltimore, 1973.

4. J. W. Macki and J. S. W. Wong, Oscillation theorems for linear second order ordinary differential equations, Proc. Amer. Math. Soc. 20 (1969), 67-72.

5. D. Willett, On the ascillatory behavior of the solutions of second onder linear differential equations, Ann. Polon. Math. 21 (1969), 175-194.

Department of Mathematics, Johns Hopkins University, Baltimore, Maryland 21218 